\title{
What Characterizes Long-term Survivors of Recurrent Ovarian Cancer? Case Report and Review of the Literature
}

\author{
ZIAD HILAL $^{1}$, BEATE SCHULTHEIS ${ }^{2}$, FRANZISKA HARTMANN ${ }^{3}$, ASKIN DOGAN ${ }^{1}$, \\ CEM CETIN $^{1}$, HARALD KRENTEL $^{4}$, SVEN SCHIERMEIER $^{5}$ and CLEMENS B. TEMPFER ${ }^{1}$ \\ Departments of ${ }^{1}$ Obstetrics and Gynecology, ${ }^{2}$ Hematology/Oncology and \\ ${ }^{3}$ Pathology, Ruhr University Bochum, Bochum, Germany; \\ ${ }^{4}$ Department of Obstetrics and Gynecology, St. Anna Hospital, Herne, Germany; \\ ${ }^{5}$ Department of Obstetrics and Gynecology, University of Witten/Herdecke, Witten, Germany
}

\begin{abstract}
Background: Women with recurrent ovarian cancer have a poor prognosis and short survival. However, some women are long-term survivors and it is unclear whether they share specific common characteristics. Case Report: We present the case of a 63-year-old woman with histologicallyproven recurrent ovarian cancer and a survival time of 16 years after the diagnosis of recurrence. She underwent initial debulking surgery in 1994, followed by 6 cycles of adjuvant chemotherapy with cisplatin and paclitaxel. After recurrent disease was diagnosed by re-laparotomy in 2000, she underwent four lines of systemic chemotherapy from 2000 to 2009 (carboplatin/paclitaxel, topotecan, etoposide/treosulfan and liposomal doxorubicin) and four lines of endocrine therapy between 2002 and 2014 (tamoxifen, goserelin, tamoxifen and exemestane). In 2014, she underwent secondary debulking surgery and was tumor-free until 2015. Upon progression, she was then started on the fifth-line of endocrine therapy, fulvestrant, which was changed to the mTOR inhibitor everolimus in June 2016. In a PUBMED literature search, 360 cases of long-term survivors of recurrent ovarian cancer (LTSROC), defined as women with survival $>5$ years after the diagnosis of recurrence, were identified with a mean postrecurrence survival time of 7.5 years. Comparing the patient and therapy details of these women, we identified common characteristics of LTSROC, i.e. young age and optimal debulking at initial surgery, a long time span between first-
\end{abstract}

Correspondence to: Clemens B. Tempfer, MD, MBA, Department of Obstetrics and Gynecology, Ruhr University Bochum, Marienhospital Herne, Hoelkeskampring 40, 44625 Herne, Germany. Tel: +49 23234991801, Fax: +49 23234993393, e-mail: clemens.tempfer@rub.de

Key Words: Ovarian cancer, recurrence, long-term survivor, chemotherapy, therapy response. line therapy and first recurrence and the combined use of optimal cytoreductive surgery and systemic chemotherapy. Conclusion: LTSROC are rare, with 360 cases described in the literature. LTSROC are characterized by young age, low tumor stage, long recurrence-free interval and combined modality treatment with optimal cytoreductive surgery and systemic chemotherapy.

Ovarian cancer is a rare disease with a life-time risk of $1.7 \%$ (1). The prognosis of women with ovarian cancer is poor, since approximately $75 \%$ of women with ovarian cancer are initially diagnosed at an advanced disease stage with the tumor having spread to the abdomen or even to more distant sites, such as the pleural space $(1,2)$. In accordance with the predominantly late stage at first presentation, ovarian cancer is the most lethal of all pelvic malignancies. High recurrence rates between 60 and $85 \%$ within five years are typical for this disease despite aggressive primary treatment with debulking surgery and systemic platinum-based chemotherapy, today combined in many cases with targeted angiogenesis inhibition with bevacizumab (1-3). Long-term survival and cure after primary therapy is possible in women with early- and late-stage disease. Long-term survivors of ovarian cancer are characterized by specific features. For example, Dao et al. identified 203 long-term survivors of ovarian cancer from the database of a multicenter research consortium (4). They found that long-term survivors of ovarian cancer generally had favorable clinical features at the time of primary therapy, including optimal surgical cytoreduction and primary platinum-sensitive disease. In accordance, Sczcesny et al. identified extensive primary surgery, platinum sensitivity, young age and good performance status as predictors of long-term survival in a cohort of 174 women from the Norwegian National Cancer Registry (5). In addition to clinical and pathological characteristics, genomic stability also seems to be a hallmark 
of long-term survival. For example, Stalberg et al. assessed genomic profiles in an integrative genetic analysis of patients with serous ovarian cancer (6). Using single nucleotide polymorphism (SNP) arrays and targeted next-generation sequencing of 26 genes, they observed an increased genomic stability, a lower proportion of somatic copy number alterations and a lower average ploidy among ovarian cancer patients with long-term survival compared to those with early cancer-specific death.

In contrast to these well-described predictors for longterm survival in the primary situation, less is known on the predictors of long-term survival in women with ovarian cancer recurrence. Ovarian cancer recurrence predominantly occurs within the abdomen and is characterized by peritoneal carcinomatosis and ascites $(1,2,4)$. Clearly, the prognosis for women with recurrent ovarian cancer is poor with a median survival time of less than 2 years $(2,3)$. It is assumed that there is no cure for women with recurrent ovarian cancer and palliative systemic chemotherapy is, therefore, the standard of care. In the recurrent situation, intravenous systemic chemotherapy with platinum compounds, taxanes, anthracyclines, gemcitabine, topotecan and trabectedine in various combinations and sequences are typically used. These regimens achieve median overall survival times after the first, second, third, fourth and fifth relapse of 17.6 (95\% confidence interval $(\mathrm{CI})=16.4-18.6$ ), 11.3 (95\% CI=10.4-12.9), 8.9 (95\% CI=0.8-9.9), 6.2 (95\% $\mathrm{CI}=5.1-7.7)$, and $5.0 \quad(95 \% \quad \mathrm{CI}=3.8-10.4) \quad$ months, respectively (2). Recent developments in the treatment of recurrent ovarian cancer include the neoangiogenesis inhibitor bevacizumab and the poly ADP ribose polymerase (PARP) inhibitor olaparib added to mono- or polychemotherapy regimens $(7,8)$. Due to the poor prognostic outlook of women with recurrent disease and median survival times between 5 and 17 months (2), women with recurrent ovarian cancer rarely survive for a long time. However, in rare cases, women do survive for a long time after having been treated with chemotherapy, endocrine therapy, secondary debulking, exenterative surgery or a combination of these modalities. There is no definition of long-term survivors of recurrent ovarian cancer (LTSROC); however, many case reports and case series of LTSROC published in the literature typically refer to women who survive for more than five years after the diagnosis of recurrence $(4,5)$.

To highlight the characteristics of LTSROC, we report the rare case of a woman with histologically-verified recurrent ovarian cancer and a survival time of 16 years after recurrence. We describe her tumor's characteristics and multiple consecutive treatment regimens. In addition, we present a literature review of case reports and case series of LTSROC and discuss common characteristics shared by these women.

\section{Case Report}

We present the case of a 63-year-old woman with histologically proven recurrent ovarian cancer and a postrecurrence survival time of 16 years. The patient was first diagnosed with ovarian cancer in January 1994 as a chance finding. At that time, she underwent hysterectomy and bilateral salpingo-oophorectomy. Subsequently, staging surgery with removal of the omentum and the pelvic and paraaortic lymph nodes was performed in March 1994. Histology showed a serous cystadenocarcinoma of the ovary with moderately differentiated tumor cells. The International Federation of Gynecology and Obstetrics (FIGO) stage was IIIC. After surgery, the patient was macroscopically tumorfree and adjuvant systemic chemotherapy with six cycles of cisplatin and paclitaxel was administered. The patient tolerated the chemotherapy well and subsequently had no evidence of disease until 2000. At that time, a rise of the tumor marker carbohydrate antigen (CA) 125 was observed and recurrent disease was confirmed by re-laparotomy. Histology showed a serous adenocarcinoma consistent with recurrence of the formerly diagnosed ovarian cancer. Then, secondary tumor debulking was performed with nonresectable residual disease left behind in the pelvic side wall and on the small bowel. Subsequently, the patient underwent four different lines of systemic chemotherapy between 2000 and 2009 , starting with re-induction chemotherapy with carboplatin and paclitaxel, followed by a monotherapy regimen with topotecan, then followed by a combination chemotherapy with etoposide and treosulfan and, finally, the patient underwent a monotherapy regimen with liposomal doxorubicin. Between 2001 and 2016, the patient also underwent four lines of endocrine therapy (tamoxifen, goserelin acetate, tamoxifen and exemestane). In April 2014, anterior exenteration was performed with removal of a bulky tumor in the pelvis, cystectomy and creation of an ileum conduit pouch and an ileostoma. In May 2014, the patient experienced a multi-level thrombosis below the level of the vena femoralis communis, treated with the anticoagulant rivaroxabane. Subsequently, in June 2014, she had acute renal failure after a renal infection with Klebsiella oxytoca. Ileostomy reversal was performed in August 2014. The patient had no evidence of disease until November 2015, when tumor progress in the pelvis and lungs was observed by computed tomography of the chest and abdomen. She was started on the fifth-line of endocrine therapy, this time with the estrogen receptor antagonist fulvestrant. In June 2016, the therapy was changed to the mTOR inhibitor everolimus due to progressive disease in the abdomen evidenced by CT. This therapy is ongoing at the time of this report with stable disease evidenced by a computed tomography of the chest and abdomen in June 2016. Figure 1 shows hematoxylineosin stains of tumor specimens sampled at the time of 


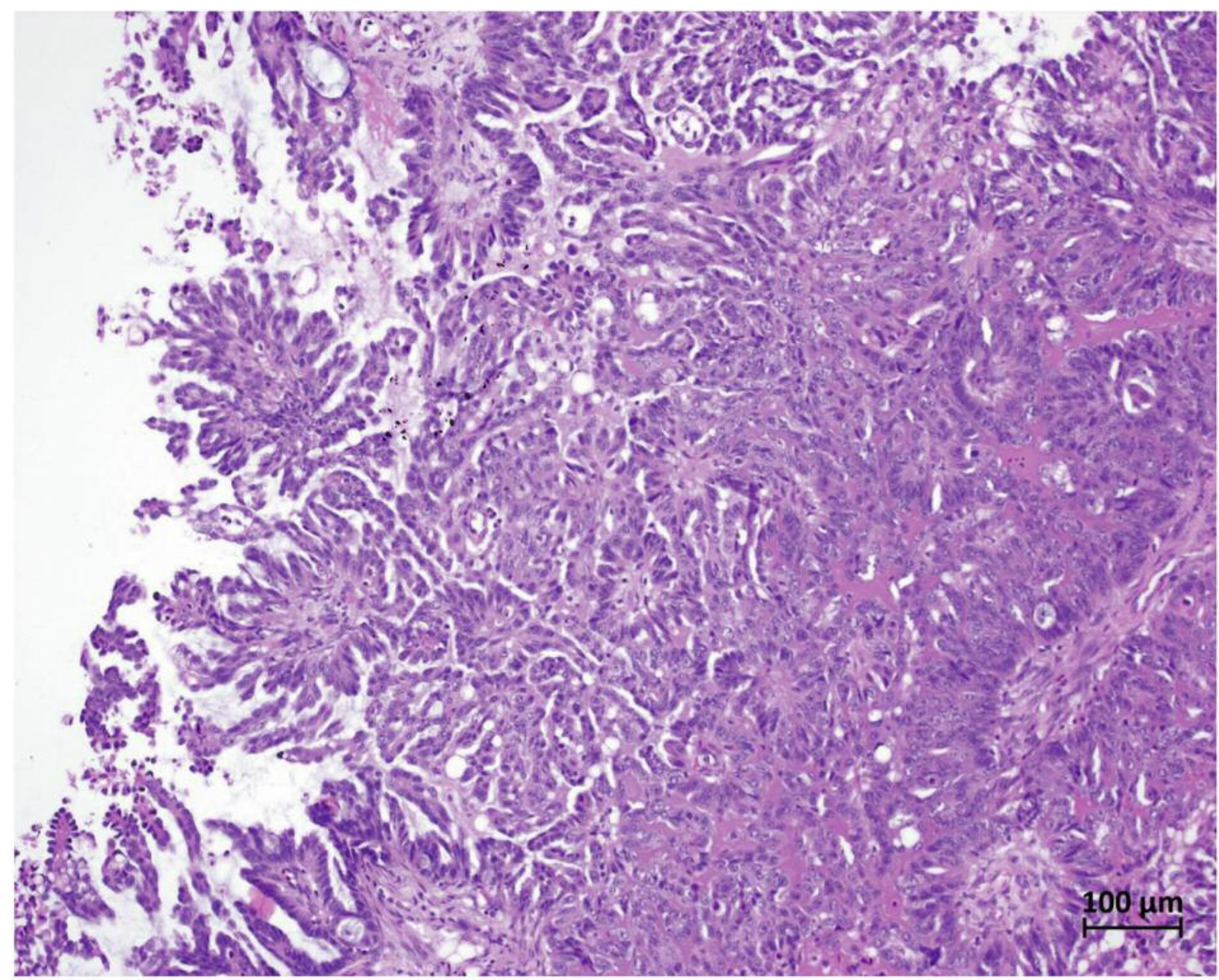

Figure 1. Hematoxylin-eosin staining of a recurrent ovarian cancer specimen sampled in 2014 demonstrating serous cystadenocarcinoma. Black bar represents $100 \mu \mathrm{m}$.

secondary tumor debulking in 2014. Figure 2 shows estrogen and progesterone receptor expression of a tumor specimen sampled in 2014 .

\section{Literature Review}

In a systematic literature search of the databases PubMed and Cochrane Central Register of Controlled Trials (search date: 16-06-2016) using the search terms "ovarian neoplasms"[MeSH Terms] OR ("ovarian"[All Fields] AND "neoplasms"[All Fields]) OR "ovarian neoplasms"[All Fields] OR ("ovarian"[All Fields] AND "cancer"[All Fields]) OR "ovarian cancer"[All Fields]) AND recurrent[All Fields] AND ("survivors"[MeSH Terms] OR "survivors"[All Fields] OR ("long"[All Fields] AND "term"[All Fields] AND "survivor"[All Fields]) OR "long term survivor"[All Fields] we identified 46 studies. After screening all abstracts, 9 studies were identified reporting on women with LTSROC, defined for the purpose of this review as a documented survival time $>5$ years after the diagnosis of ovarian cancer recurrence (4, 9-16). Studies not reporting on long-term survival after ovarian cancer recurrence and studies reporting on women with borderline ovarian tumors or germ cell tumors of the ovary were excluded. After cross-searching the references of the 9 studies identified using this strategy, 8 additional studies were identified (17-24). In summary, 17 studies were included in this review. Figure 3 shows a diagram of the literature search. Table I shows the study characteristics and outcomes of the patients described in the 17 studies identified describing 360 cases of LTSROC. Specifically, 237 (66\%) of LTSROC experienced one recurrence, whereas 2 and $\geq 3$ recurrences were experienced by $52(15 \%)$ and $71(20 \%)$ of LTSROC, respectively. The mean time of post-recurrence overall survival was $7.5 \pm 6.9$ years. 


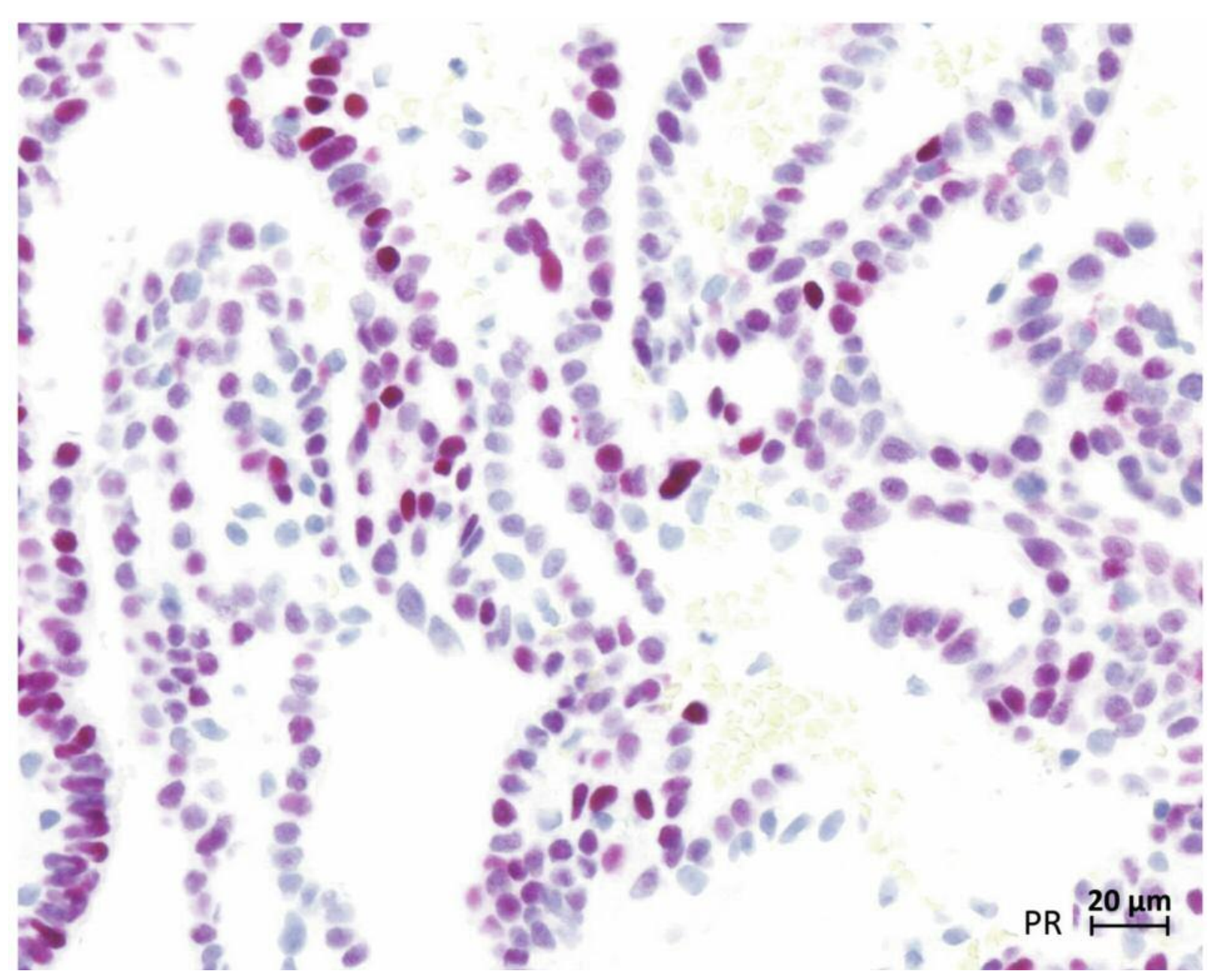

Figure 2. Immunohistochemical staining of recurrent ovarian cancer specimens sampled in 2014 demonstrating the expression of estrogen (upper panel) and progesterone receptors (lower panel). Black bars representing $20 \mu \mathrm{m}$. ER, Estrogen receptor; PR, progesterone receptor.

The largest series of LTSROC was published by Dao et al. (4). In a large series of 203 ovarian cancer survivors with a history of high-grade serous adenocarcinoma, the authors identified 100 women with a survival time of at least 10 years post-recurrence (4). They found that LTSROC generally had favorable clinical features, including optimal initial cytoreductive surgery (CRS) and primary platinum-sensitive disease. Of note, there was no uniform pattern of time to recurrence in the 100 women described in this cohort study. Some of the patients recurred early and continued to have longterm survival, whereas other patients recurred late and also had long-term survival after recurrence. Both situations were common and equally represented in this cohort of LTSROC.

Optimal surgical cytoreduction at the time of recurrence with no residual tumor left behind was described as a reliable predictor of post-recurrence LTSROC in four studies $(7,10$,
12, 18). For example, in a series of 87 women with recurrence after early-stage ovarian cancer, Gadducci et al. identified two independent predictors of post recurrence survival, i.e. young patient age and successful surgical cytoreduction at the time of recurrence (7). Likewise, Tentes et al. stressed the fact that complete cytoreduction at the time of recurrence best predicted overall survival in their analysis of 29 women with recurrent ovarian cancer who underwent cytoreductive surgery (CRS) combined with perioperative intraperitoneal chemotherapy (10). Also, Petrillo et al., assessing 70 women with platinum-sensitive recurrence, found that optimal cytoreduction, in these cases combined with hyperthermic intraperitoneal chemotherapy (HIPEC), resulted in favorable 5- and 7-year survival rates (18).

Radiotherapy in combination with CRS or systemic chemotherapy has been described as an effective therapeutic 


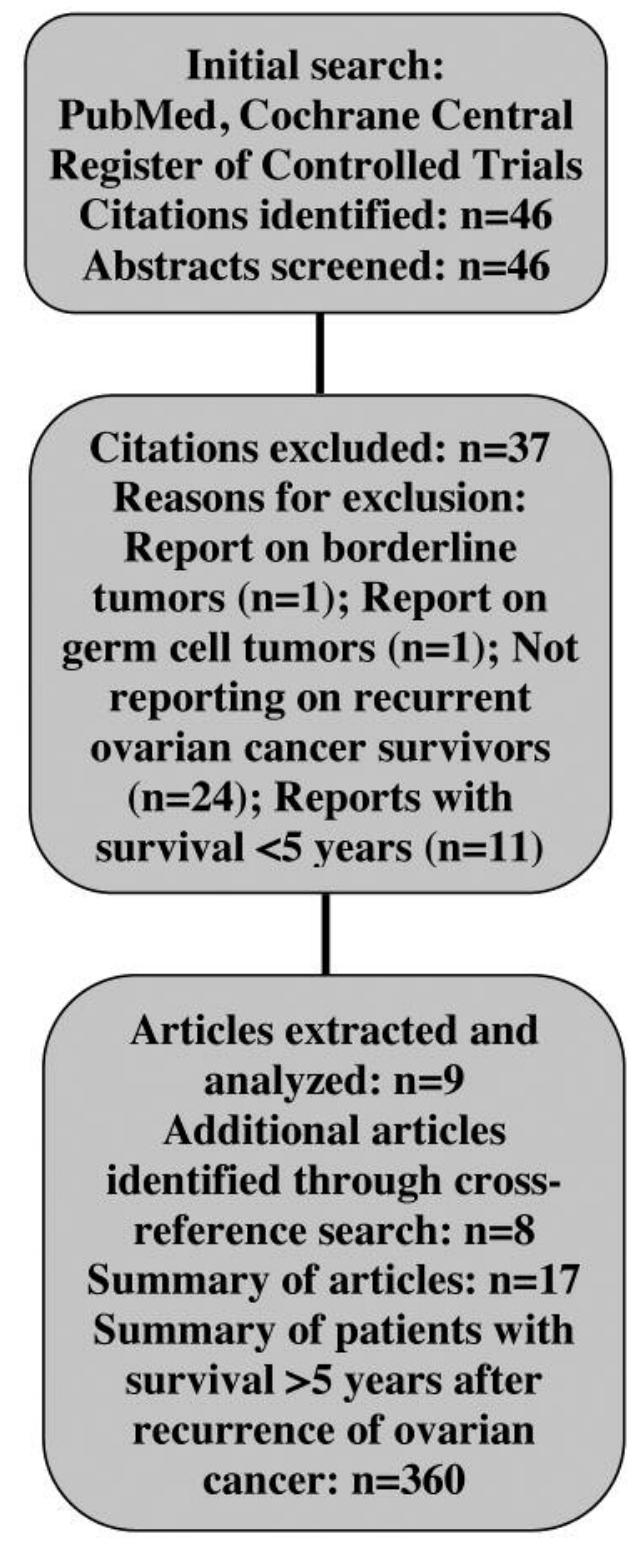

Figure 3. Flow diagram of the literature search algorithm.

strategy in 4 studies $(15,16,17,21)$. Firat et al. used selective radiotherapy in the management of 28 women with recurrent ovarian cancer involving the vagina or rectum achieving long-term survival $>5$ years in 4 of them (15). Albuquerque et al. described 8 LTSROC among a group of 27 women with localized extraperitoneal recurrences from epithelial ovarian cancer who received tumor volumedirected involved field radiation therapy at the time of recurrence (17). Eight of 14 LTSROC described by Iwase et al. received radiotherapy in combination with surgery and/or systemic chemotherapy (21). Finally, Martinez-Monge et al. reported one case of LTSROC after intraoperative radiotherapy combined with $\mathrm{CRS}$ and external beam irradiation, as well as systemic chemotherapy (16). Taken together, radiotherapy at the time of ovarian cancer recurrence was effectively used in 57/360 (16\%) LTSROC.

Systemic chemotherapy is the standard of care for women with recurrent ovarian cancer $(1,2)$. In accordance with that, in $14 / 17(82 \%)$ studies and in 328/360 (91\%) patients identified in our literature review, systemic chemotherapy was used as the sole treatment modality or as part of the therapeutic concept. In 15/16 studies, CRS was also performed and in 4/16 studies some form of radiotherapy (RXT) was added to CRS and systemic chemotherapy. Therefore, one characteristic feature of LTSROC seems to be the combination of therapeutic modalities with systemic chemotherapy, CRS and RXT being the most often cited combinations.

Only a minority of the reports identified in this literature review discussed endocrine therapy. For example, ChudeckaGlaz et al. described one patient with 9 years of survival postrecurrence solely treated with a gonadotropin releasing hormone agonist (11). Likewise, our own patient described in the present case report successfully underwent and responded to five consecutive lines of endocrine therapy, including tamoxifen, goserelin acetate, exemestane and fulvestrant.

\section{Discussion}

In this case report and review of the literature, we describe a woman with histologically-proven recurrent ovarian cancer and a post-recurrence survival time of 16 years. During that time, the patient underwent a series of treatments, including five lines of systemic chemotherapy, two CRS and five lines of endocrine therapy. In a systematic literature search, we identified 17 studies with 360 patients identified as LTSROC. Common characteristics shared by LTSROC were young age and optimal debulking at the time of initial surgery, a long recurrence-free interval and the combined use of optimal cytoreductive surgery and systemic chemotherapy. In a minority of LTSROC, durable responses to endocrine therapies were noted.

Many treatments and treatment combinations for LTSROC were used in the 17 studies analyzed in this review. The individual management strategies are outlined in Table I demonstrating that there is no specific therapy that is characteristic for LTSROC. However, a combination of CRS, systemic chemotherapy and -in some studies- radiotherapy has been used in the majority of successfully treated patients. When looking for common characteristics of LTSROC shared by a majority of women, we identified the following parameters:

i) optimal debulking at initial surgery,

ii) young patient age at initial surgery, 
Table I. Clinical studies describing cases of women with long-term survival of $>5$ years after ovarian cancer recurrence.

\begin{tabular}{|c|c|c|c|c|c|c|c|}
\hline Author & Year & $\begin{array}{l}\text { Number of } \\
\text { cases (n) }\end{array}$ & $\begin{array}{c}\text { One } / \text { two } / \geq \text { three } \\
\text { recurrences } \\
\text { (n) }\end{array}$ & $\begin{array}{c}\text { Initial } \\
\text { FIGO stage }\end{array}$ & Histology & $\begin{array}{l}\text { Treatment } \\
\text { regimens }\end{array}$ & $\begin{array}{l}\text { Survival time } \\
\text { after recurrence- } \\
\text { years (n) }\end{array}$ \\
\hline Martinez-Monge et al. & 1993 & 1 & $1 /-/-$ & - & EOC & CRS, RXT, CHXT & $7(1)$ \\
\hline Eltabbakh et al. & 1998 & 11 & $6 / 5 /-$ & I-IV & EOC & CRS, CHXT-M & $9(11)$ \\
\hline Firat et al. & 2001 & 4 & $1 / 3 /$ & I-III & EOC & CRS, CHXT, RXT & $10(1) ; 19(1) ; 27(1) ; 6(1)$ \\
\hline Chan et al. & 2004 & 2 & $-/-/ 2$ & III & EOC, SAC & CRS, CHXT, ET & $7.6(1) ; 10.5(1)$ \\
\hline Jha et al. & 2005 & 1 & $1 /-/-$ & I & AS & Surgery, CHXT & $5(1)$ \\
\hline Fleisch et al. & 2007 & 1 & 1/-/- & III & EOC & CRS, CHXT & $5(1)$ \\
\hline Legge et al. & 2008 & 16 & $-/ 10 / 6$ & II-IV & EOC & Surgery, CHXT & $5(16)$ \\
\hline Chudecka-Glaz et al. & 2009 & 1 & $1 /-/-$ & - & EOC & $\mathrm{ET}$ & $9(1)$ \\
\hline Tentes et al. & 2010 & 14 & $14 /-/-$ & - & - & CRS, ICHXT & $5(14)$ \\
\hline Kajiyama et al. & 2012 & 102 & $102 /-/-$ & II-IV & EOC & CRS, CHXT & $5(102)$ \\
\hline Gaducci et al. & 2013 & 30 & $30 /-/-$ & II-IV & EOC & CHXT, CRS, RXT & $5(4) ; 7(26)$ \\
\hline Bacalbasa et al. & 2015 & 4 & $1 / 1 / 2$ & I-III & EOC & CRS, CHXT & $5.5(3) ; 10(1)$ \\
\hline Bentivegna et al. & 2015 & 13 & $12 / 1 /-$ & I-II & EOC & Surgery, CRS & $15.5(11) ; 24(1) ; 19(1)$ \\
\hline Iwase et al. & 2015 & 14 & 14/-/- & I-III & EOC & CRS, CHXT, RXT & $>5(14)$ \\
\hline Dao et al. & 2016 & 100 & $21 / 19 / 60$ & I - IV & EOC & CHXT, CRS & $10(100)$ \\
\hline Albuquerque et al. & 2016 & 8 & $8 /-/-$ & - & EOC & CRS, RXT & $5(3) ; 10(5)$ \\
\hline Petrillo et al. & 2016 & 37 & 24/13/- & I-IV & EOC & CRS, HIPEC, CHXT & $5(6) ; 7(31)$ \\
\hline Hilal et al. & 2016 & 1 & $-/-/ 1$ & III & EOC & CRS, CHXT, ET & $16(1)$ \\
\hline Pooled Analysis & - & 360 & $\begin{array}{c}237(66 \%) / 52 \\
(14 \%) / 71(20 \%)\end{array}$ & - & - & - & Mean $7.5(360)$ \\
\hline
\end{tabular}

n, Number of cases; EOC, epithelial ovarian cancer; CRS, cytoreductive surgery; RXT, radiotherapy; CHXT-M, maintenance chemotherapy; CHXT, chemotherapy; SAC, serous adenocarcinoma; AS, angiosarcoma; ET, endocrine therapy; ICHXT, intraoperative chemotherapy; HIPEC, hyperthermic intraperitoneal chemotherapy.

iii) a long time span between the end of the first-line therapy and the first recurrence, and

iv) successful application of multi-modality treatment with optimal cytoreduction and systemic chemotherapy and/or radiotherapy.

In contrast to our expectation, endocrine therapy did not play an important role in the treatment of LTSROC. We could only identify two cases of LTSROC effectively treated with endocrine therapy in this literature review. Therefore, based on the literature search performed, endocrine therapy clearly is not a typical treatment effectively used in LTSROC.

Based on personal experience, women surviving ovarian cancer recurrence for more than 5 years are a rare clinical finding. Therefore, it was surprising to identify the high number of 360 cases in the literature. Although still rare, these cases demonstrate that the chance of LTSROC is considerable and that the assumption of recurrent ovarian cancer being definitively incurable should be seen critically. Obviously, there is a small subset of women with certain characteristics -as identified in our literature review- for whom LTSROC is possible. These women have tumors occurring early in life and are well debulkable and platinumsensitive. In these women, aggressive treatment strategies at the time of recurrence, including CRS combined with radiotherapy and systemic chemotherapy, may be justified.

\section{Conclusion}

In conclusion, we describe the case of a woman with a survival time of 16 years after ovarian cancer recurrence. In a systematic review of the literature, we identified 360 LTSROC with specific characteristics shared by a majority of them. Based on the data available in the literature, we conclude that LTSROC is rare but possible and characterized by biological variables, such as young age at the time of initial diagnosis and management-dependent variables, such as optimal debulking at initial surgery, optimal CRS at the time of recurrence and application of multi-modality treatments combining CRS, radiotherapy and systemic chemotherapy.

\section{Conflicts of Interest}

The Authors declare that they have no conflict of interest.

\section{References}

1 Foley OW, Rauh-Hain JA and del Carmen MG: Epithelial ovarian cancer: An update on treatment. Oncology 27(4): 288294, 2003.

2 Fung-Kee-Fung M, Oliver T, Elit L, Oza A, Hirte HW and Bryson P: Optimal chemotherapy treatment for women with recurrent ovarian cancer. Curr Oncol 14(5): 195-208, 2007. 
3 Hanker LC, Loibl S and Burchardi N: The impact of second to sixth line therapy on survival of relapsed ovarian cancer after primary taxane/platinum-based therapy. Ann Oncol 23(10): 2605-2612, 2012.

4 Dao F, Schlappe BA, Tseng J, Lester J, Nick AM and Lutgendorf SK: Characteristics of 10-year survivors of high-grade serous ovarian carcinoma. Gynecol Oncol 141(2): 260-263, 2016.

5 Szczesny W, Vistad I, Kaern J, Nakling J, Tropé C and Paulsen $\mathrm{T}$ : Impact of hospital type and treatment on long-term survival among patients with FIGO Stage IIIC epithelial ovarian cancer: follow-up through two recurrences and three treatment lines in search for predictors for survival. Eur J Gynaecol Oncol 37(3): 305-311, 2016.

6 Stalberg K, Crona J, Razmara M, Taslica D, Skogseid B and Stalberg P: An integrative genomic analysis of formalin fixed paraffin-embedded archived serous ovarian carcinoma comparing long-term and short-term survivors. Int $\mathbf{J}$ Gynecol Cancer 26(6): 1027-1032, 2016.

7 Gadducci A and Guerrieri ME: parp inhibitors in epithelial ovarian cancer: State of art and perspectives of clinical research. Anticancer Res 36(5): 2055-2064, 2016.

8 McClung EC and Wenham RM: Profile of bevacizumab in the treatment of platinum-resistant ovarian cancer: current perspectives. Int J Womens Health 8: 59-75, 2016.

9 Gadducci A, Cosio S, Zola P, Sostegni B, Fuso L and Sartori E: Prognostic factors and clinical outcome of patients with recurrent early-stage epithelial ovarian cancer: an Italian multicenter retrospective study. Int J Gynecol Cancer 23(3): 461468, 2013.

10 Tentes AA, Korakianitis OS, Kakolyris S, Kyziridis D, Veliovits D, Karagiozoglou C, Sgouridou E and Moustakas K: Cytoreductive surgery and perioperative intraperitoneal chemotherapy in recurrent ovarian cancer. Tumori 96(3): 411416, 2010.

11 Chudecka-Głaz A and Rzepka-Górska I: Favorable effects of long-term therapy with gonadoliberin analogues in three patients with advanced and recurrent ovarian cancer. Eur J Gynaecol Oncol 30(5): 589-591, 2009.

12 Fleisch MC, Pantke P, Beckmann MW, Schnuerch HG, Ackermann R, Grimm MO, Bender HG and Dall P: Predictors for long-term survival after interdisciplinary salvage surgery for advanced or recurrent gynecologic cancers. J Surg Oncol 95(6): 476-484, 2007.

13 Jha S, Chan KK, Poole CJ and Rollason TP: Pregnancy following recurrent angiosarcoma of the ovary--a case report and review of literature. Gynecol Oncol 97(3): 935-937, 2005.

14 Chan JK, Loizzi V, Manetta A and Berman ML: Oral altretamine used as salvage therapy in recurrent ovarian cancer. Gynecol Oncol 92(1): 368-371, 2004

15 Firat S and Erickson B: Selective irradiation for the treatment of recurrent ovarian carcinoma involving the vagina or rectum. Gynecol Oncol 80(2): 213-220, 2001.
16 Martínez Monge R, Jurado M, Azinovic I, Aristu JJ, Tangco E, Viera JC, Berián JM and Calvo FA: Intraoperative radiotherapy in recurrent gynecological cancer. Radiother Oncol 28(2): 127$133,1993$.

17 Albuquerque K, Patel M, Liotta M, Harkenrider M, Guo R, Small W Jr, and Ronald P: Long-term benefit of tumor volumedirected involved field radiation therapy in the management of recurrent ovarian cancer. Int J Gynecol Cancer 26(4): 655-660, 2016.

18 Petrillo M, De Iaco P, Cianci S, Perrone M, Costantini B, Ronsini C, Scambia G and Fagotti A: Long-term survival for platinum-sensitive recurrent ovarian cancer patients treated with secondary cytoreductive surgery plus hyperthermic intraperitoneal chemotherapy (HIPEC). Ann Surg Oncol 23(5): 1660-1665, 2016.

19 Bacalbaşa N, Balescu I, Dima S and Popescu I: Long-term survivors after liver resection for ovarian cancer liver metastases. Anticancer Res 35(12): 6919-6923, 2015.

20 Bentivegna E, Fruscio R, Roussin S, Ceppi L, Satoh T, Kajiyama $\mathrm{H}$, Uzan C, Colombo N, Gouy S and Morice P: Long-term follow-up of patients with an isolated ovarian recurrence after conservative treatment of epithelial ovarian cancer: Review of the results of an international multicenter study comprising 545 patients. Fertil Steril 104(5): 1319-1324, 2015.

21 Iwase H, Takada T, Iitsuka C, Nomura H, Abe A, Taniguchi T, Sakamoto K, Takizawa K and Takeshima N: Clinical features of long-term survivors of recurrent epithelial ovarian cancer. Int $\mathrm{J}$ Clin Oncol 20(1): 143-149, 2015.

22 Eltabbakh GH, Piver MS, Hempling RE, Recio FO and Blumenson LE: Prolonged disease-free survival by maintenance chemotherapy among patients with recurrent platinum-sensitive ovarian cancer. Gynecol Oncol 71(2): 190-195, 1998.

23 Kajiyama H, Shibata K, Mizuno M, Umezu T, Suzuki S, Yamamoto E, Fujiwara S, Kawai M, Nagasaka T and Kikkawa F: Long-term clinical outcome of patients with recurrent epithelial ovarian carcinoma: Is it the same for each histological type? Int J Gynecol Cancer 22(3): 394-399, 2012.

24 Legge F, Petrillo M, Adamo V, Pisconti S, Scambia G and Ferrandina G: Epithelial ovarian cancer relapsing as isolated lymph node disease: Natural history and clinical outcome. BMC Cancer 8: 367, 2008.

25 Chan JK, Tian C, Teoh D, Monk BJ, Herzog T, Kapp DS and Bell J: Survival after recurrence in early-stage high-risk epithelial ovarian cancer: A Gynecologic Oncology Group study. Gynecol Oncol 116(3): 307-311, 2010.
Received July 31, 2016

Revised August 17, 2016

Accepted August 18, 2016 